MSC 14J60, 14D20, 14M27

UDC $512.722+512.723$

\title{
ON A NEW COMPACTIFICATION OF MODULI OF VECTOR BUNDLES ON A SURFACE, IV: NONREDUCED MODULI
}

\author{
Nadezda V. TIMOFEEVA \\ Yaroslavl' State University \\ Sovetskaya str. 14, 150000 Yaroslavl', Russia \\ e-mail: ntimofeeva@list.ru
}

\begin{abstract}
The construction for nonreduced projective moduli scheme of semistable admissible pairs is performed. We establish the relation of this moduli scheme with reduced moduli scheme built up in the previous article and prove that nonreduced moduli scheme contains an open subscheme which is isomorphic to moduli scheme of semistable vector bundles.

Bibliography: 10 items.
\end{abstract}

Keywords: moduli space, semistable coherent sheaves, moduli functor, algebraic surface.

\section{Introduction}

We construct the moduli scheme for the functor of admissible semistable pairs $((\widetilde{S}, \widetilde{L}), \widetilde{E})$ introduced in the previous articles [1]-[5] of the author. There we passed to the reduction of the intermediate scheme used in the construction. This yields in the reduced scheme $\widetilde{M}$ with no relation to (non)reducedness of the functor of moduli. The aim of the present article is to give precise sense to this situation and to construct (possibly) nonreduced moduli whenever the functor of families is nonreduced.

We start with

- smooth irreducible projective algebraic surface $S$ over an algebraically closed field $k$ of zero characteristic;

- ample invertible $\mathcal{O}_{S}$-sheaf $L$ which is fixed and called polarization of the surface $S$;

\footnotetext{
${ }^{0}$ This work was partially supported by the Institute of Mathematics "Simion Stoilow" of the Romanian Academy (IMAR) (partnership IMAR - BITDEFENDER), during author's visit as invited professor on June - July 2011.
} 
- positive integer $r$ and polynomial $r p_{E}(n)$ with rational coefficients to be interpreted as rank and Hilbert polynomial of coherent sheaves on $S$. Hilbert polynomial is compute with respect to $L$. of

We work with admissible semistable pairs $((\widetilde{S}, \widetilde{L}), \widetilde{E})$. Any such pair consists

- admissible scheme of view $\widetilde{S}=\operatorname{Proj} \bigoplus(I[t]+(t))^{s} /(t)^{s+1}$ where $I \subset \mathcal{O}_{S}$ is ideal sheaf of colength equal to $l \leq C$. Positive integer $C$ depends on coefficients of the Hilbert polynomial $r p_{E}(n)$ (see $\S 3$ ). The case $\widetilde{S} \cong S$ is also considered. The structure morphism $\sigma: \widetilde{S} \rightarrow S$ is called canonical morphism of the scheme $\widetilde{S}$. It is identity isomorphism when $\widetilde{S} \cong S$;

- ample invertible $\mathcal{O}_{\widetilde{S}}$-sheaf $\widetilde{L}=L^{m} \otimes \sigma^{-1} I \cdot \mathcal{O}_{\widetilde{S}}$. It is called the distinguished polarization for the scheme $\widetilde{S}$. The number $m$ can be chosen common for all $\widetilde{S}$ (claim 193 ). Fix this $m$ and redenote $L^{m}$ by $L$ so that $\widetilde{L}=$ $L \otimes \sigma^{-1} I \cdot \mathcal{O}_{\widetilde{S}}$

- semistable locally free $\mathcal{O}_{\widetilde{S}}$-sheaf $\widetilde{E}$ of rank $r$ with Hilbert polynomial $r p_{E}(n)$ if it is compute with respect to the distinguished polarization $\widetilde{L}$. The sheaf $\widetilde{E}$ is required to satisfy the quasi-ideality condition (see (1.4) below).

It is clear that admissible scheme of the view $\widetilde{S}=$ $\operatorname{Proj} \bigoplus_{s \geq 0}(I[t]+(t))^{s} /\left(t^{s+1}\right)$ can be naturally represented as a union of irreducible components $\widetilde{S}=\bigcup_{i \geq 0} \widetilde{S}_{i}$ where the main component $\widetilde{S}_{0}=\operatorname{Proj} \bigoplus_{s \geq 0}(I)^{s}$ is the blowup of the surface $S$ in the sheaf of ideals $I$ and for $i>0 \widetilde{S}_{i}$ are irreducible additional components $\bigcup_{i>0} \widetilde{S}_{i}$. As it is shown in [4], in this case the additional component can have a structure of nonreduced scheme. Obviously, admissible scheme consists of a single component $\widetilde{S}=\widetilde{S}_{0}$ if and only if it is isomorphic to the initial surface $S$.

The restriction $\sigma_{0}=\left.\sigma\right|_{\widetilde{S}_{0}}: \widetilde{S}_{0} \rightarrow S$ of the canonical morphism $\sigma$ onto the main component $\widetilde{S}_{0}$ is a blowup morphism.

Following [6, ch. 2, sect. 2.2] we recall some definitions. Let $\mathcal{C}$ be a category, $\mathcal{C}^{o}$ its opposite, $\mathcal{C}^{\prime}=\mathcal{F}$ unct $\left(\mathcal{C}^{o}\right.$, Sets $)$ - a category of functors to the category of sets. By Yoneda lemma, the functor $\mathcal{C} \rightarrow \mathcal{C}^{\prime}: F \mapsto\left(\underline{F}: X \mapsto \operatorname{Hom}_{\mathcal{C}}(X, F)\right)$ includes $\mathcal{C}$ as a full subcategory in $\mathcal{C}^{\prime}$.

Definition 1. [6, ch. 2, definition 2.2.1] The functor $\mathfrak{f} \in \mathcal{O} b \mathcal{C}^{\prime}$ is corepresented by the object $F \in \mathcal{O b C}$, if there exist $\mathcal{C}^{\prime}$-morphism $\psi: \mathfrak{f} \rightarrow \underline{F}$ such that any morphism $\psi^{\prime}: \mathfrak{f} \rightarrow \underline{F}^{\prime}$ factors through the unique morphism $\omega: \underline{F} \rightarrow \underline{F}^{\prime}$. 
Let $T$ be a scheme over the field $k$. Consider families of semistable pairs

$$
\mathfrak{F}_{T}=\left\{\begin{array}{l}
\pi: \mathbb{F} \rightarrow T, \widetilde{\mathbb{L}} \in \text { Pic } \mathbb{F}, \forall t \in T \widetilde{L}_{t}=\left.\widetilde{\mathbb{L}}\right|_{\pi^{-1}(t)} \text { is ample; } \\
\left(\pi^{-1}(t), \widetilde{L}_{t}\right) \text { admissible scheme with distinguished } \\
\text { polarisation; } \\
\widetilde{\mathbb{E}}-\text { locally free } \mathcal{O}_{\mathbb{F}}-\text { sheaf; } \\
\left.\left.\chi\left(\widetilde{\mathbb{E}} \otimes \widetilde{\mathbb{L}}^{m}\right)\right|_{\pi^{-1}(t)}\right)=r p_{E}(m) ; \\
\left(\left(\pi^{-1}(t), \widetilde{L}_{t}\right),\left.\widetilde{\mathbb{E}}\right|_{\pi^{-1}(t)}\right)-(\text { semi) stable pair }
\end{array}\right\}
$$

and a functor $\mathfrak{f}:\left(\text { Schemes }_{k}\right)^{o} \rightarrow($ Sets $)$ from the category of $k$-schemes to the category of sets. This functor assigns to any scheme $T$ the set of equivalence classes $\left(\mathfrak{F}_{T} / \sim\right)$.

The equivalence relation $\sim$ is defined as follows. Families $((\pi: \mathbb{F} \rightarrow T, \widetilde{\mathbb{L}}), \widetilde{\mathbb{E}})$ and $\left(\left(\pi^{\prime}: \mathbb{F}^{\prime} \rightarrow T, \widetilde{\mathbb{L}}^{\prime}\right), \widetilde{\mathbb{E}}^{\prime}\right)$ of the class $\mathfrak{F}$ are said to be equivalent (notation: $\left.((\pi: \mathbb{F} \rightarrow T, \widetilde{\mathbb{L}}), \widetilde{\mathbb{E}}) \sim\left(\left(\pi^{\prime}: \mathbb{F}^{\prime} \rightarrow T, \widetilde{\mathbb{L}}^{\prime}\right), \widetilde{\mathbb{E}}^{\prime}\right)\right)$ if

1) there is an isomorphism $\iota: \mathbb{F} \stackrel{\sim}{\longrightarrow} \mathbb{F}^{\prime}$ such that the diagram

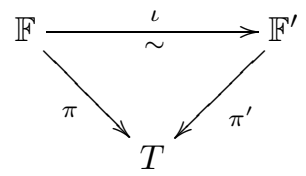

commutes.

2) There are linear bundles $L^{\prime}, L^{\prime \prime}$ on $T$ such that $\iota^{*} \widetilde{\mathbb{E}}^{\prime}=\widetilde{\mathbb{E}} \otimes \pi^{*} L^{\prime}, \iota^{*} \widetilde{\mathbb{L}}^{\prime}=$ $\widetilde{\mathbb{L}} \otimes \pi^{*} L^{\prime \prime}$.

Definition 2. The scheme $\widetilde{M}$ is a coarse moduli space of the functor $\mathfrak{f}$ if $\mathfrak{f}$ is corepresented by the scheme $\widetilde{M}$.

The result of the present article is contained in the following theorem.

Theorem 1. The functor $\mathfrak{f}$ has a coarse moduli space $\widetilde{M}$ which is a projective Noetherian algebraic scheme of finite type. The scheme $\widetilde{M}$ contains an open subscheme $\widetilde{M}_{0}$ isomorphic to open subscheme $\bar{M}_{0}$ of locally free sheaves in the Gieseker - Maruyama scheme $\bar{M}$ corresponding to the same data $r, p_{E}(n)$.

All the reasoning of the present paper is applicable to any Hilbert polynomial with no relation to the value of discriminant as well as to the number and geometry of irreducible components in the corresponding Gieseker - Maruyama scheme. In general (reducible) case the theorem provides the existence of the coarse moduli space for any maximal (under inclusion) irreducible substack in $\amalg\left(\mathfrak{F}_{T} / \sim\right)$ if it contains pairs $\left(\left(\pi^{-1}(t), \widetilde{L}_{t}\right),\left.\widetilde{\mathbb{E}}\right|_{\pi^{-1}(t)}\right)$ such that $\left(\pi^{-1}(t), \widetilde{L}_{t}\right) \cong$ $(S, L)$. Such pairs are called $S$-pairs. We mean under $\widetilde{M}$ the moduli space of a substack containing semistable $S$-pairs.

The article is organized as follows. $\S 1$ comprises necessary additions and corrections for the article [5. In $\S 2$ we recall the well-known definitions and 
results concerning (non)reducedness in functorial setting. We prove the proposition about interplay between the reduction of the functor and of its moduli scheme. $\S 3$ is devoted to the construction moduli scheme $\widetilde{M}$ in the category of all $k$-schemes. There are two critical points: boundedness of families to parameterize (proposition 4 \$3) and quasi-projectivity of the corresponding scheme (proposition 5 \$3). §4 contains the necessary material about subfunctors and moduli subspaces and completes the proof of theorem 1 .

\section{Comments for [5]}

In this section we consider the corrections and additions for the construction of moduli scheme $\widetilde{M}$ done in [5]. All the results hold; the changes concern some proofs. All reasonings and results of the article [5] are done for the functor $\mathfrak{f}$ on full subcategory of reduced schemes $\left(R S c h_{k}\right)^{o}$ in $\left(S c h e m e s_{k}\right)^{o}$, although this fact was not reflected explicitly in the text. Respectively, we mention under $\bar{M}$ the reduced scheme corresponding to Gieseker - Maruyama scheme.

Also in the definition for the equivalence of families $((\pi: \mathbb{F} \rightarrow T, \widetilde{\mathbb{L}}), \widetilde{\mathbb{E}})$ $\left(\left(\pi^{\prime}: \mathbb{F}^{\prime} \rightarrow T, \widetilde{\widetilde{L}^{\prime}}\right), \widetilde{\mathbb{E}^{\prime}}\right)$ (the requirement 2 following the diagram $\left.(0.1)\right)$ must read as in Introduction of the present article.

In the formulas for the sheaf $L_{E}$ in $\S 2$ (preceding the diagram (2.2)) there are misprints; these formulas must read as $L_{E}=\bigwedge^{r}\left(E \otimes L^{m}\right)=L^{m} \otimes \operatorname{det} E$ and (below) $L_{E}=L^{m} \otimes c_{1}$.

Below we give the correction for the requirement of quasi-ideality for sheaves $\widetilde{E}$, and corrections in $\S 7$.

The end of $\S 1$ must read as follows.

The behavior of vector bundles $\widetilde{E}$ on additional components $\widetilde{S}_{i} \subset \widetilde{S}, i>0$ is given by the following easy computation. Standard exact triple (1.1)

$$
0 \rightarrow E \rightarrow E^{\vee \vee} \rightarrow \varkappa \rightarrow 0
$$

is taken by the functor of direct image $\sigma_{i}^{*}$ to the exact sequence

$$
\cdots \rightarrow \mathcal{T}_{\text {or }_{1}}^{\sigma_{i}^{-1} \mathcal{O}_{S}}\left(\sigma^{-1} \varkappa, \mathcal{O}_{\widetilde{S}_{i}}\right) \rightarrow \sigma_{i}^{*} E \rightarrow \sigma_{i}^{*} E^{\vee \vee} \rightarrow \sigma_{i}^{*} \varkappa \rightarrow 0 .
$$

In appropriate neighborhood $U \subset S$ of the support Supp $\varkappa$ the locally free sheaf $\left.E^{\vee \vee}\right|_{U}$ can be replaced by its local trivialization $\mathcal{O}_{U}^{\oplus r}$. Then the exact sequence (1.2) takes the form

$$
\cdots \rightarrow \mathcal{T o r}_{1}^{\sigma_{i}^{-1} \mathcal{O}_{S}}\left(\sigma^{-1} \varkappa, \mathcal{O}_{\widetilde{S}_{i}}\right) \rightarrow \sigma_{i}^{*} E \rightarrow \sigma_{i}^{*} \mathcal{O}_{U}^{\oplus r} \rightarrow \sigma_{i}^{*} \varkappa \rightarrow 0
$$

Consequently, for $\widetilde{E}_{i}=\sigma^{*} E /\left.\operatorname{tors}\right|_{\widetilde{S}_{i}}=\sigma_{i}^{*} E / \operatorname{tors}_{i}$ we have

$$
\cdots \rightarrow \sigma_{i}^{*} E / \text { tors }_{i} \rightarrow \sigma_{i}^{*} \mathcal{O}_{U}^{\oplus r} \rightarrow \sigma_{i}^{*} \varkappa \rightarrow 0,
$$

where subsheaf of torsion tors ${ }_{i}$ on (possibly, nonreduced) scheme $\widetilde{S}_{i}$ is defined as before and tors $i=$ tors $\left.\right|_{\widetilde{S}_{i}}$. Dots on the left hand side mean the terms which violate exactness. These terms are not obliged to have positive codimension in $\widetilde{S}_{i}$. 
Example 1. Let $\varkappa=k_{x}$, then $\widetilde{S}$ consists of two reduced components: $\widetilde{S}_{0}$ is a surface obtained by blowing up of the reduced point $x$ on the surface $S$, and $\widetilde{S}_{1} \cong$ $\mathbb{P}^{2}$. The morphism $\sigma_{1}$ is constant morphism $\sigma_{1}: \mathbb{P}^{2} \rightarrow x$. Then $\sigma_{1}^{*} \varkappa=\sigma_{1}^{*} k_{x}=$ $\mathcal{O}_{\mathbb{P}^{2}}$, and easy counting of ranks leads to $\operatorname{rank} \operatorname{ker}\left(\sigma_{1}^{*} E / \operatorname{tors}_{1} \rightarrow \mathcal{O}_{\widetilde{S}_{1}}^{\oplus r}\right)=1$.

Since the sheaf $\varkappa$ is supported in a finite collection of points, then the morphism $\mathcal{O}_{U}^{\oplus r} \rightarrow \varkappa$ can be replaced by the morphism $\mathcal{O}_{S}^{\oplus r} \rightarrow \varkappa$.

Let $q_{0}: \mathcal{O}_{S}^{\oplus r} \rightarrow \varkappa$ be a morphism induced by the exact sequence (1.1). Then we have

$$
\widetilde{E}_{i}=\sigma_{i}^{*} \operatorname{ker} q_{0} / \text { tors } .
$$

According to Proposition 1 [5], for all semistable coherent sheaves $E$ with fixed Hilbert polynomial $r p_{E}(m)$ all sheaves $\widetilde{E}_{i}$ on additional components $\widetilde{S}_{i}$ can be described by relations of the form (1.4) for appropriate $q_{0} \in \coprod_{l \leq c_{2}} \operatorname{Quot}^{l} \mathcal{O}_{S}^{\oplus r}$.

The exact sequence (1.3) and the relation (1.4) provide right requirement for quasi-ideality. This requirement must be of use instead of (1.5) and (1.6) in [5] whenever it is involved: in the definition of $S$-(semi)stability (Definition 6, $\S 5)$, and in proofs of Proposition 10 (§6) and of Lemma 3 (§8).

Definition 3. S-stable (respectively, semistable) pair $((\widetilde{S}, \widetilde{L}), \widetilde{E})$ is the following data:

- $\widetilde{S}=\bigcup_{i \geq 0} \widetilde{S}_{i}$ admissible scheme, $\sigma: \widetilde{S} \rightarrow S$ canonical morphism, $\sigma_{i}: \widetilde{S}_{i} \rightarrow$ $S$ its restrictions on components $\widetilde{S}_{i}, i \geq 0$;

- $\widetilde{E}$ vector bundle on the scheme $\widetilde{S}$;

- $\widetilde{L} \in$ Pic $\widetilde{S}$ distinguished polarization;

such that

- $\chi\left(\widetilde{E} \otimes \widetilde{L}^{n}\right)=r p_{E}(n)$;

- the sheaf $\widetilde{E}$ on the scheme $\widetilde{S}$ is Gieseker-stable (respectively, Giesekersemistable), i.e. for any proper subsheaf $\widetilde{F} \subset \widetilde{E}$ for $n \gg 0$

$$
\begin{aligned}
& \frac{h^{0}\left(\widetilde{F} \otimes \widetilde{L}^{n}\right)}{\operatorname{rank} F}<\frac{h^{0}\left(\widetilde{E} \otimes \widetilde{L}^{n}\right)}{\operatorname{rank} E}, \\
&\text { (respectively, } \left.\frac{h^{0}\left(\widetilde{F} \otimes \widetilde{L}^{n}\right)}{\operatorname{rank} F} \leq \frac{h^{0}\left(\widetilde{E} \otimes \widetilde{L}^{n}\right)}{\operatorname{rank} E}\right) ;
\end{aligned}
$$

- on each additional component $\widetilde{S}_{i}, i>0$, the sheaf $\widetilde{E}_{i}:=\left.\widetilde{E}\right|_{\widetilde{S}_{i}}$ is quasi-ideal, i.e. it has a description of the form (1.4) for some $q_{0} \in \bigsqcup_{l \leq c_{2}} \operatorname{Quot}^{l} \bigoplus^{r} \mathcal{O}_{S}$. 
The end of the proof of Proposition 10 [5] (following the inclusion of inverse images of trivial sheaves) after the replacement takes the following form.

There is a commutative triangle

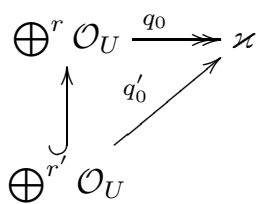

where the morphism $q_{0}^{\prime}$ is defined as composite map. Applying the functor of direct image $\sigma^{*}$ and restrictions on each additional component we have

$$
\begin{aligned}
\widetilde{E}_{i} & =\sigma_{i}^{*} \operatorname{ker} q_{0} / \operatorname{tors}_{i}, \\
\widetilde{F}_{i}=\sigma^{*} F /\left.\operatorname{tors}\right|_{\widetilde{S}_{i}} & =\sigma_{i}^{*} \operatorname{ker} q_{0}^{\prime} / \operatorname{tors}{ }_{i} .
\end{aligned}
$$

This completes the proof.

In the proof of Lemma 3 [5] the formula

$$
\left.\widetilde{E}_{i}^{\prime}\right|_{\text {add }}=\left.\operatorname{ker} \sigma^{*}\left(\oplus^{r} \mathcal{O}_{S} \rightarrow \varkappa\right)\right|_{\text {add }}
$$

must be replaced by the formula

$$
\left.\widetilde{E}_{i}^{\prime}\right|_{\text {add }}=\left.\sigma^{*} \operatorname{ker}\left(\oplus^{r} \mathcal{O}_{S} \rightarrow \varkappa\right)\right|_{\text {add }} / \text { tors } .
$$

The proof of Proposition 13 [5, §7] is wrong: although the number of isomorphism classes of Artinian quotient algebras with bounded length for the ring $k[x, y]$ is finite, the set of equivalence classes of morphisms $\mathcal{O}_{S} \rightarrow \varkappa$ for length $\varkappa \leq l_{0}$ is infinite. For example, let $\varkappa$ be the algebra of dual numbers corresponding to nonreduced subscheme of length 2 ("infinitesimal tangent vector"). Choice of various directions of this vector provides infinite collection of non-equivalent morphisms $\mathcal{O}_{S} \rightarrow \varkappa$.

Proposition 13 itself, as well as Definition 14 (together with Remarks 6 and 7) can be (and must be) removed without any loss for the results of the article.

Proposition 14 must be preceded by the proposition which has in the text number 15 and must be formulated with respect to $\diamond$-product $\bar{S}_{*}=\widetilde{S} \diamond \widetilde{S}_{g r}$ :

Proposition 1. If $\bar{\sigma}: \bar{S}_{*} \rightarrow S$ is $\diamond$-product in the monoid $\diamond[E]$, then for any $E \in[E]$ images $\bar{F}_{i}:=\bar{\sigma}^{*} F_{i} /$ tors of sheaves $F_{i}$ in Jordan - Hölder filtration are locally free and $\bar{\sigma}^{*} g r_{i}(E)=\bar{F}_{i} / \bar{F}_{i-1}$.

The proof is preserved literally.

In the proof of Proposition 27 [5] minimal resolution must be replaced by $\diamond$-product $\bar{S}_{*}=\widetilde{S} \diamond \widetilde{S}_{g r}$.

To construct the scheme $\widetilde{M}$ we need a smooth resolution $\xi: Q^{\prime} \rightarrow Q \quad Q \subset$ Quot $^{r p_{E}(t)}\left(V \otimes L^{-m}\right)$, where $V=H^{0}\left(S, E \otimes L^{m}\right)$. Then the standard resolution of the family of semistable coherent sheaves with the base $Q^{\prime}$ is performed. This 
leads to a birational morphism $\phi: \widetilde{Q} \rightarrow Q^{\prime}$. To proceed further one needs equivariance of morphisms $\xi$ and $\phi$. Equivariance in of use when GIT-quotient $\mu(\widetilde{Q}) / P G L(V)=\widetilde{M}$ is constructed.

Equivariance of smooth resolution can be achieved by replacement of resolution due to $\mathrm{H}$. Hironaka by the canonical equivariant resolution done by O. Villamayor and collaborators (cf., for example, $[$ ]). The construction performed in [5], operates with base schemes with reduced scheme structures. In particular, the scheme $Q$ is mentioned to be reduced scheme. Then consider each component of the scheme $\operatorname{Quot}^{r p_{E}(t)}\left(V \otimes L^{-m}\right)$ as reduced scheme, and for appropriate $l \gg 0$ consider closed $G L(V)$-equivariant immersion

$$
i_{l}: \operatorname{Quot}^{r p_{E}(t)}\left(V \otimes L^{-m}\right) \hookrightarrow G\left(V \otimes H^{0}\left(L^{l-m}\right), r p_{E}(l)\right) .
$$

Then there is an induced closed equivariant immersion of each component of the closure $\bar{Q}$ of the subscheme $Q$ in the scheme $\operatorname{Quot}^{r p_{E}(t)}\left(V \otimes L^{-m}\right)$, into the same variety $G\left(V \otimes H^{0}\left(L^{l-m}\right), r p_{E}(l)\right)$. Then we can think of $\bar{Q}$ as reduced equidimensional subscheme in $G\left(V \otimes H^{0}\left(L^{l-m}\right), r p_{E}(l)\right)$.

The situation mentioned above allows to apply the result of 8 , theorem $2.4]$ what provides required $G L(V)$-equivariant resolution. The algorithm of equivariant resolution consists of a sequence of blowups in invariant closed subschemes. Let $X$ be a scheme acted upon by an algebraic group $G \alpha: G \times X \rightarrow X$, and $\xi: \widehat{X} \rightarrow X$ be an equivariant morphism. Convince that the action $\alpha$ induces an action $\widehat{\alpha}: G \times \widehat{X} \rightarrow \widehat{X}$ in the sense that the commutative square

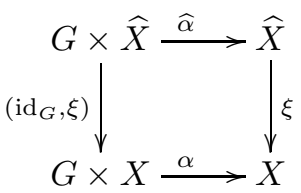

is Cartesian.

Let $T$ be a scheme, $f: T \rightarrow G \times X$ and $h: T \rightarrow \widehat{X}$ its morphisms such that $\xi \circ h=\alpha \circ f$. We denote as inv $: G \rightarrow G$ the morphism inverting elements of the group $G$, and as $p_{G}: G \times X \rightarrow G$ the projection on the first factor. Then the morphism $\varphi: T \rightarrow G \times \widehat{X}$ is uniquely defined by the formula $\varphi=$ $\left(p_{G} \circ f, \widehat{\alpha} \circ\left(\right.\right.$ inv $\left.\left.\circ p_{G} \circ f, h\right)\right)$ what proves the universality of the square (1).

On the next step of the construction we perform a standard resolution of the family of semistable coherent sheaves $\mathbb{E}$ on the $Q$-based trivial family of surfaces $Q \times S$. Note that choice of $Q$ as smooth resolution of subscheme in the Grothendieck's scheme Quot is not unique: if there is a $P G L(V)$-equivariant resolution $Q$ then for further usage any blowup of $Q$ in $P G L(V)$-equivariant smooth subscheme will fit as well as $Q$. In this case the construction of standard resolution described in 2, 3, and applied to different schemes $Q$, leads to different families $\widetilde{Q}, \pi: \widetilde{\Sigma} \rightarrow \widetilde{Q}, \widetilde{\mathbb{E}}$. Although non-uniquely definedness of scheme $\widetilde{Q}$ does not affect the final result of the construction.

When the moduli scheme $\widetilde{M}$ is built up the morphism $\mu: \widetilde{Q} \rightarrow \operatorname{Hilb}^{P(t)} G(V, r)$ and $P G L(V)$-invariant subscheme $\mu(\widetilde{Q})$ are involved. The subscheme yields in 
GIT-quotient $\widetilde{M}=\mu(\widetilde{Q}) / P G L(V)$. By [5, proposition 18] the subscheme $\mu(\widetilde{Q})$ corresponds to the subset of those closed points in $\operatorname{Hilb}^{P(t)} G(V, r)$ which are defined by objects of parametrization (admissible semistable pairs). This is the reason why the subscheme $\mu(\widetilde{Q})$ does not depend of the choice of resolution $Q$ and of the scheme $\widetilde{Q}$ which is constructed by $Q$.

Definition 4. The collection $(\widetilde{Q}, \pi: \widetilde{\Sigma} \rightarrow \widetilde{Q}, \widetilde{\mathbb{L}}, \widetilde{\mathbb{E}})$ where $\pi$ is a flat morphism of schemes, $\widetilde{\mathbb{E}}$ locally free sheaf flat over $\widetilde{Q}, \widetilde{\mathbb{L}}$ invertible sheaf which provides distinguished polarizations on fibres of $\pi$, is called a standard resolution of the family $(Q, p: Q \times S \rightarrow Q, \mathbb{L}, \mathbb{E})$ of coherent semistable sheaves if

- there are birational morphisms $\phi: \widetilde{Q} \rightarrow Q$ and $\Phi: \widetilde{\Sigma} \rightarrow Q \times S$ fitting into the commutative diagram

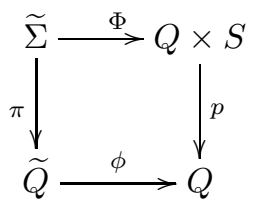

- morphisms $\phi$ and $\Phi$ become isomorphisms when restricted on subschemes $\widetilde{Q}_{0}=\phi^{-1} Q_{0}$ and $\widetilde{\Sigma}_{0}=\Phi^{-1}\left(Q_{0} \times S\right)$;

- for each closed point $\widetilde{q} \in \widetilde{Q}$ the corresponding member $\left(\left(\pi^{-1}(\widetilde{q}),\left.\widetilde{\mathbb{L}}\right|_{\pi^{-1}(\widetilde{q})}\right)\right.$, $\left.\left.\widetilde{\mathbb{E}}\right|_{\pi^{-1}(\widetilde{q})}\right)$ of the family is semistable admissible pair;

- there is a descent rule: $\left(\Phi_{*} \widetilde{\mathbb{E}}\right)^{\vee \vee}=\mathbb{E}$.

Let the scheme $Q$ is supplied with an action $\beta: G \times Q \rightarrow Q$ of algebraic group $G$.

Definition 5. The standard resolution $\phi: \widetilde{Q} \rightarrow Q$ is called equivariant if there is an action $\alpha: G \times \widetilde{Q} \rightarrow \widetilde{Q}$ of algebraic group $G$ on the scheme $\widetilde{Q}$ such that the diagram

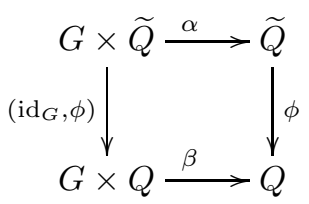

commutes.

To analyze GIT-stability of points of the subscheme $\mu(\widetilde{Q})$ and to apply 5 , $\S 9]$ we can replace (arbitrary) standard resolution $\widetilde{Q}$ by $P G L(V)$-equivariant standard resolution.

We point out the equivariant standard resolution $\widetilde{Q}^{\prime}$ such that actions of $G=P G L(V)$ on schemes $Q$ and $\mu(\widetilde{Q})$ are $\widetilde{Q}^{\prime}$-concordant in the sense of [5, $\S 9$ ]. 
To construct this resolution form a product of actions $\alpha$ and $\beta$ in the following fashion:

$$
\alpha \times \beta: G \times \mu(\widetilde{Q}) \times Q \rightarrow \mu(\widetilde{Q}) \times Q:(g, \widetilde{q}, q) \mapsto(\alpha(g, \widetilde{q}), \beta(g, q)) .
$$

This is true action of the group $G$ on the product of schemes $\mu(\widetilde{Q}) \times Q$. Now consider a locally closed subscheme ("the diagonal") $\left(\mu\left(\phi^{-1}\right), i\right): Q_{0} \hookrightarrow \mu(\widetilde{Q}) \times Q$ in this product. The diagonal is defined on closed points by the correspondence $q \mapsto\left(\mu\left(\phi^{-1}(q)\right), q\right)$. Here $i: Q_{0} \hookrightarrow Q$ means the open immersion and we take into account that $\left.\phi\right|_{\phi^{-1}\left(Q_{0}\right)}: \widetilde{Q}_{0} \rightarrow Q_{0}$ is an isomorphism. Note that the image $\left(\mu\left(\phi^{-1}\right), i\right)\left(Q_{0}\right)$ is $G$-invariant subscheme with respect to the action $\alpha \times \beta$. Define a subscheme $\widetilde{Q}^{\prime}$ as a closure $\widetilde{Q}^{\prime}:=\overline{\left(\mu\left(\phi^{-1}\right), i\right)\left(Q_{0}\right)}$. It is also $G$-invariant under the action $\alpha \times \beta$. Then we can define an action $\widetilde{\alpha}: G \times \widetilde{Q}^{\prime} \rightarrow \widetilde{Q}^{\prime}$ by restriction of the product $\alpha \times \beta$ on the invariant subscheme $\widetilde{Q}^{\prime}: \widetilde{\alpha}:=\alpha \times\left.\beta\right|_{G \times \widetilde{Q}^{\prime}}$. Note that the scheme $\widetilde{Q}^{\prime}$ has two surjective morphisms of projections on factors as defined by the diagram

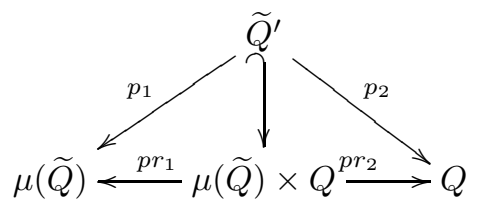

For $\widetilde{Q}^{\prime}$-concordance of actions $\alpha$ and $\beta$ it is sufficient to confirm that both left hand side and right hand side squares of the commutative diagram

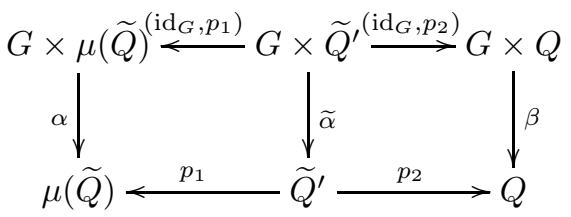

are Cartesian.

We perform the proof of the universality for the left hand side square because the manipulations for the right hand side square are analogous. Let $T$ be a scheme, $f: T \rightarrow G \times \mu(\widetilde{Q})$ and $h: T \rightarrow \widetilde{Q}^{\prime}$ are morphisms making the square

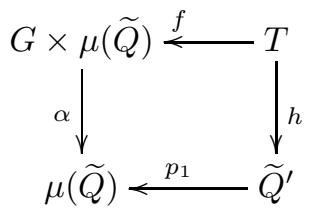

to commute. Let $t \in T$ be a closed point, $f(t)=(g, \widetilde{q}) \in G \times \mu(\widetilde{Q})$ and $h(t)=\widetilde{q}^{\prime}$ its images under morphisms $f$ and $h$ respectively. Let also $\alpha(g, \widetilde{q})=g \widetilde{q}$. By commutativity of the left hand side square in (1.5) $p_{1}(g \widetilde{q})=\widetilde{q}^{\prime}$. Then 
$\left(\operatorname{id}_{G}, p_{1}\right)(g, \widetilde{q})=\left(g, p_{1}(\widetilde{q})\right)=\left(g, g^{-1} \widetilde{q}\right)$. Define a map $\varphi: T \rightarrow G \times \widetilde{Q}^{\prime}$ by the correspondence $t \mapsto\left(g, g^{-1} \widetilde{q}^{\prime}\right)$. It is clear that the map $\varphi$ is uniquely defined. Denoting by the symbol inv : $G \rightarrow G$ the inverting morphism in the group $G$ and by the symbol $p_{G}: G \times \mu(\widetilde{Q}) \rightarrow G$ projection to the factor we define a morphism $\varphi$ by the formula

$$
\varphi=\left(p_{G} \circ f, \widetilde{\alpha} \circ\left(\operatorname{inv} \circ p_{G} \circ f, h\right)\right) .
$$

Author presents her deepest apologies for mistakes and gaps in the text of the article [5].

\section{The reduction of moduli functor and its mod- uli scheme}

First recall some definitions [9, ch. 1, sect. 4.5].

Let $X=\left(X, \mathcal{O}_{X}\right)$ be a scheme and let $\mathcal{N} i l_{X} \subset \mathcal{O}_{X}$ be the nilradical of the structure sheaf $\mathcal{O}_{X}$. Then the quotient sheaf $\mathcal{O}_{X} / \mathcal{N} i l_{X}$ will be denoted as $\mathcal{O}_{X_{\text {red }}}$. The scheme $X_{\text {red }}:=\left(X, \mathcal{O}_{X_{\text {red }}}\right)$ will be referred to as a reduction of the scheme $\left(X, \mathcal{O}_{X}\right)$. It is clear that the reduction of the scheme $\left(X, \mathcal{O}_{X}\right)$ is homeomorphic to the scheme $X$ as Zariski topological space and that it is canonically embedded subscheme in $X$ [9, ch. 1, corollaire 4.5.2]. If $\rho: X_{\text {red }} \hookrightarrow$ $X$ be the morphism of immersion then the corresponding sheaf morphism is the morphism onto the quotient sheaf $\rho^{\sharp}: \mathcal{O}_{X} \rightarrow \mathcal{O}_{X_{\text {red }}}$.

Proposition 2. [9, proposition 4.5.10] For any scheme morphism $f: X \rightarrow Y$ there is a canonically defined morphism of the corresponding reduced schemes $f_{\text {red }}: X_{\text {red }} \rightarrow Y_{\text {red }}$ making the diagram

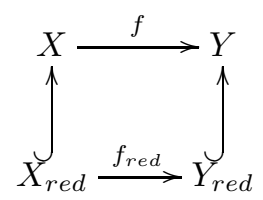

to commute.

This morphism will be called the reduction of the morphism $f$.

Now consider any functor of the following view

$$
\mathfrak{f}:\left(\text { Schemes }_{k}\right)^{o} \rightarrow \text { Sets }
$$

from the category of schemes over the field $k$ to the category of sets. The functor of our interest attaches to any scheme $T \in \mathrm{ObSchemes}{ }_{k}$ the set $\mathfrak{F}_{T}$ of $T$-based families of objects of some prescribed type. Let $\left(R S c h_{k}\right)^{\circ}$ be a subcategory formed by reduced schemes and their morphisms in $\left(\text { Schemes }_{k}\right)^{\circ}$. Then we have a natural inclusion (as a full subcategory) $\mathfrak{i}:\left(R S c h_{k}\right)^{o} \hookrightarrow\left(\text { Schemes }_{k}\right)^{\circ}$ and a functor of reduction $\mathfrak{r e d}:\left(\text { Schemes }_{k}\right)^{o} \rightarrow\left(R S c h_{k}\right)^{o}$ such that the composite $\mathfrak{r} \mathfrak{d} \circ \mathfrak{i}$ is identity functor on $\left(R S c h_{k}\right)^{\circ}$. 
We call the restriction $\mathfrak{f}_{\text {red }}:=\left.\mathfrak{f}\right|_{\left(R S c h_{k}\right)^{\circ}}$ of the functor $\mathfrak{f}$ onto subcategory $\left(R S c h_{k}\right)^{o}$ as reduction of the functor $\mathfrak{f}$. It is natural to say that the functor $\mathfrak{f}$ is reduced if it factors as $\mathfrak{f}=\mathfrak{f}_{\text {red }} \circ \mathfrak{r e d}$.

The same can be done if the corresponding functor of moduli

$$
\mathfrak{f} / \sim:\left(\text { Schemes }_{k}\right)^{o} \rightarrow \text { Sets }
$$

is considered. This means that it attaches to any scheme $T$ the set of classes of $T$-based families $\mathfrak{F}_{T} / \sim$ with respect to some appropriate equivalence relation $\sim$.

Proposition 3. Let $\mathfrak{f}$ be corepresented by a scheme $M$. Then its reduction $\mathfrak{f}_{\text {red }}$ is corepresented by the reduction $M_{\text {red }}$ of the scheme $M$.

Proof. Since $\mathfrak{f}$ is corepresented by the scheme $M$ then there exist a morphism $\psi$ : $\mathfrak{f} \rightarrow(X \mapsto \operatorname{Hom}(X, M))$ such that any morphism $\psi^{\prime}: \mathfrak{f} \rightarrow\left(X \mapsto \operatorname{Hom}\left(X, F^{\prime}\right)\right)$ factors through the unique morphism

$$
\omega:(X \mapsto \operatorname{Hom}(X, M)) \rightarrow\left(X \mapsto \operatorname{Hom}\left(X, F^{\prime}\right)\right) .
$$

Passing to reductions of $\mathfrak{f}, X, F^{\prime}, M$ one gets the required.

\section{A nonreduced moduli scheme for $\mathfrak{f}$}

In this section we construct (possibly) nonreduced moduli scheme for admissible S-semistable pairs. Afterwards we prove that this scheme is quasi-projective. In the situation of interest this is enough to conclude that the nonreduced moduli scheme is projective scheme.

To construct moduli space one encounters two problems: to examine the boundedness of families of the interest and to apply geometric invariant theory in his situation. In our case due to the special choice of distinguished polarizations $\widetilde{L}$ Hilbert polynomials both for admissible schemes $\widetilde{S}$ and for semistable vector bundles $\widetilde{E}$ remain constant over the base.

Claim 1. There exist a (common for all semistable admissible pairs) integer $m \gg 0$ such that the invertible sheaf $\widetilde{L}=L^{m} \otimes \sigma^{-1} I \cdot \mathcal{O}_{\widetilde{S}}$ is very ample.

Now we explain why some finite (may be big enough) integer $m$ can be sufficient for all schemes $\widetilde{S}$. Isomorphy classes of schemes $\widetilde{S}=\operatorname{Proj} \bigoplus_{s \geq 0}(I[t]+$ $(t))^{s} /(t)^{s+1}$ are enumerated by 0 -dimensional subschemes in $S$ corresponding to the ideals $I$.

It is clear from the construction of the schemes $\widetilde{S}, 4$ that lengths of these 0 -dimensional subschemes are bounded by the function of coefficients of the Hilbert polynomial of sheaves of our interest: $l \leq C$. Indeed, the sheaf of ideals $I$ is defined as 0 -th Fitting ideal sheaf $\mathcal{F} i t t^{0} \mathcal{E} x t^{2}\left(\varkappa, \mathcal{O}_{S}\right)$ for $\varkappa$ being the quotient sheaf $E^{\vee \vee} / E$. It is compute by coherent semistable torsion-free $\mathcal{O}_{S}$-sheaf $E$ with Hilbert polynomial $r p_{E}(n)$ and defines its resolution of singularity. Note that 
length $\mathcal{E} x t^{2}\left(\varkappa, \mathcal{O}_{S}\right)=$ length $\varkappa$ and the last length is bounded from above by the second Chern class length $\varkappa \leq c_{2}$ of sheaves of interest. Note that there is only finite collection of isomorphy classes of Artinian quotient $k[x, y]_{(x, y)}$-modules of $k[x, y]_{(x, y)}^{\oplus r}$ of the fixed length. Here $k[x, y]_{(x, y)}$ means the localization of the ring $k[x, y]$ in the maximal ideal $(x, y)$. So we come to the conclusion that the set of colengths colength $\mathcal{F} i t t^{0} \mathcal{E} x t^{2}\left(\varkappa, \mathcal{O}_{S}\right)$ is finite and hence bounded from above.

Then all isomorphy classes of $\widetilde{S} \neq S$ can be depicted as points of the following scheme of finite type: $\coprod_{l=1}^{C} \operatorname{Hilb}^{l} S$

Convention 1. Fix this $m$ and redenote $L^{m}$ by $L$ so that $\widetilde{L}=L \otimes \sigma^{-1} I \cdot \mathcal{O}_{\widetilde{S}}$ as was mentioned in the introduction. Also for any flat family of schemes of the class $\widetilde{S}$ with distinguished polarizations $\widetilde{L}$, let $\widetilde{\mathbb{L}}$ be an invertible sheaf which is very ample relatively to $T$. It is assumed tacitly to give distinguished polarization on each fibre when restricted onto this fibre.

Let $X$ be a projective scheme over a field $k$ and let $\mathcal{O}(1)$ be a very ample line bundle.

Definition 6. [6, Definition 1.7.5] A family of isomorphism classes of coherent sheaves on $X$ is bounded if there is a $k$-scheme $S$ of finite type and a coherent $\mathcal{O}_{S \times X}$-sheaf $F$ such that the given family is contained in the set

$$
\left\{\left.F\right|_{\text {Spec } k_{s} \times X} \mid s \text { a closed point in } S\right\} .
$$

Theorem 2. [6, Theorem 3.3.7] Let $f: X \rightarrow S$ be a projective morphism of schemes of finite type over $k$ and let $\mathcal{O}_{X}(1)$ be an $f$-ample line bundle. Let $P$ be a polynomial of degree $d$, and let $\mu_{0}$ be a rational number. Then the family of purely d-dimensional sheaves on the fibres of $f$ with Hilbert polynomial $P$ and maximal slope $\widehat{\mu}_{\max } \leq \mu_{0}$ is bounded. In particular, the family of semistable sheaves with Hilbert polynomial $P$ is bounded.

Proposition 4. Family of admissible semistable pairs with fixed rank $r$ and Hilbert polynomial $r p_{E}(n)$ is bounded.

Proof. We can consider any fixed integer $n \geq 1$ so as all admissible schemes $\widetilde{S}$ are immersed into projective space $\mathbb{P}=P\left(H^{0}\left(\widetilde{S}, \widetilde{L}^{n}\right)\right)$ as closed subschemes and all projective spaces $\mathbb{P}=P\left(H^{0}\left(\widetilde{S}, \widetilde{L}^{n}\right)\right)$ are isomorphic by the construction. Define $\chi(n)$ as Hilbert polynomial of any subscheme $j: \widetilde{S} \hookrightarrow \mathbb{P}$ i.e. $\chi(n):=$ $\chi\left(j^{*} \mathcal{O}_{\mathbb{P}}(n)\right)$. Then consider corresponding Hilbert scheme $\mathbb{H}:=\mathrm{Hilb}^{\chi(n)} \mathbb{P}$ and universal subscheme $\mathbb{U}:=\operatorname{Univ}^{\chi(n)} \mathbb{P}$. Hilbert scheme $\mathbb{H}$ is (projective) scheme of finite type over $k$ and the structure morphism $\pi: \mathbb{U} \rightarrow \mathbb{H}$ is (flat) morphism of finite type. Hence the scheme $\mathbb{U}$ is of finite type over $k$.

Then we can consider families of semistable (not necessarily locally free) coherent sheaves on fibres of the morphism $\pi$. Semistability is understood in usual sense due to Gieseker, without requirement of quasi-ideality. Sheaves are considered to have Hilbert polynomial equal to $r p_{E}(n)$ when it is compute with respect to the polarization $\widetilde{L}$. By theorem 2 the set of semistable coherent sheaves with 
fixed Hilbert polynomial on fibres of the morphism $\pi$ is bounded. This set is equal to the set of all pairs $((\widetilde{S}, \widetilde{L}), \widetilde{E})$ where $(\widetilde{S}, \widetilde{L})$ be a 2 -dimensional projective polarized scheme such that its polarization $\widetilde{L}$ induces closed immersion $j: \widetilde{S} \hookrightarrow \mathbb{P}$ of $\widetilde{S}$ as a subscheme with Hilbert polynomial $\chi(n) . \widetilde{E}$ is Giesekersemistable coherent sheaf with Hilbert polynomial equal to $r p_{E}(n)$.

Since admissible semistable pairs form a subset in the set of all pairs with Gieseker-semistable coherent sheaves $\widetilde{E}$, this completes the proof of boundedness for families of admissible semistable pairs.

By proposition 4 there is (common for all semistable torsion-free sheaves $\widetilde{E}$ ) integer $m \gg 0$ such that the sheaves $\widetilde{E} \otimes \widetilde{L}^{m}$ are globally generated and for all $\widetilde{E}$ the vector spaces of global sections $H^{0}\left(\widetilde{S}, \widetilde{E} \otimes \widetilde{L}^{m}\right)$ are isomorphic to $k$-vector space $V$ of dimension $r p_{E}(m)$. This $V$ will be fixed from now.

Now we can turn to the Grassmannian variety $G(V, r)$ to perform what was done in $[5]$ for reduced case. Each pair $((\widetilde{S}, \widetilde{L}), \widetilde{E})$ with semistable locally free sheaf $\widetilde{E}$ provides a closed immersion $j: \widetilde{S} \hookrightarrow G(V, r)$. Let $\mathcal{O}_{G(V, r)}(1)$ is positive generator of the group $\operatorname{Pic} G(V, r), P(n):=\chi\left(j^{*} \mathcal{O}_{G(V, r)}(n)\right)$ be the Hilbert polynomial of closed subscheme $j(\widetilde{S})$. We form Hilbert scheme Hilb ${ }^{P(n)} G(V, r)$ and subscheme $H$ formed by all admissible semistable pairs. This subscheme is defined by scheme-theoretic images of all possible bases $T$ of families of S-semistable admissible pairs under induced morphisms to Hilbert scheme Hilb ${ }^{P(n)} G(V, r)$. Since we put no restriction to $T$ to be reduced then it is clear that $H$ can be nonreduced.

Now consider a connected component $H_{0}$ of $H$ containing $\mu(\widetilde{Q})$ mentioned in [5]. Indeed $\mu(\widetilde{Q})$ is the reduction of $H_{0}$.

Proposition 5. $H_{0}$ is quasi-projective subscheme in $\mathrm{Hilb}^{P(t)} G(V, r)$.

Proof. To prove the quasi-projectivity of $H_{0}$ we need some topological result from EGA [9, ch. 1, proposition 4.5.14]. In particular this proposition says that the scheme morphism $f: X \rightarrow Y$ is open (resp. closed, homeomorphic onto its image) if and only if the same holds for its reduction $f_{\text {red }}$.

Consider locally closed immersion $\mu(\widetilde{Q}) \hookrightarrow \operatorname{Hilb}^{P(n)} G(V, r)$. The Hilbert scheme Hilb ${ }^{P(n)} G(V, r)$ is projective $k$-scheme of finite type and $\mu(\widetilde{Q})$ is quasiprojective subscheme in it. The scheme $H_{0}$ is another subscheme in $\operatorname{Hilb}^{P(n)} G(V, r)$ such that $\left(H_{0}\right)_{\text {red }}=\mu(\widetilde{Q})$. To convince that $H_{0}$ is quasi-projective, form scheme-theoretic closures $\overline{\mu(\widetilde{Q})}$ and $\bar{H}_{0}$ for $\mu(\widetilde{Q})$ and $H_{0}$ respectively. Both closures are taken in Hilb ${ }^{P(n)} G(V, r)$. Since subschemes $\overline{\mu(\widetilde{Q})}$ and $\bar{H}_{0}$ are closed in the projective scheme they are projective. By the construction $\left(\bar{H}_{0}\right)_{\text {red }}=\overline{\mu(\widetilde{Q})}$. Now consider immersions $i: H_{0} \hookrightarrow \overline{H_{0}}$ and $i_{\text {red }}: \mu(\widetilde{Q}) \hookrightarrow \overline{\mu(\widetilde{Q})}$. By $[9$, ch. 1 , proposition 4.5.14] since $i_{\text {red }}$ is open then $i$ is also open. This implies that $H_{0}$ is quasi-projective scheme.

The group $P G L(V)$ acts upon the Grassmann variety $G(V, r)$ by linear transformations of the vector space $V$ and it acts in an induced fashion upon 
the product Hilb ${ }^{P(n)} G(V, r) \times G(V, r)$. The subscheme $H_{0} \subset \operatorname{Hilb}^{P(n)} G(V, r)$ is $P G L(V)$-invariant.

Proposition 6. There exists GIT-quotient $\widetilde{M}=H_{0} / / P G L(V)$ which is moduli scheme for the functor $\mathfrak{f}$. The scheme $\widetilde{M}$ is a projective algebraic Noetherian scheme of finite type.

Proof. The scheme $H_{0}$ is acted upon by the same algebraic group $P G L(V)$ as its reduction $\mu(\widetilde{Q})$ and geometric invariant theory is also applicable.

Let $\mathcal{S}$ be the universal quotient bundle on the Grassmannian $G(V . r)$, as usually $\mathcal{O}_{G(V, r)}(1)$ is the positive generator in its Picard group. We use following notations for projections of the universal subscheme Hilb ${ }^{P(n)} G(V, r) \stackrel{\pi}{\longleftarrow}$ Univ $^{P(n)} G(V, r) \stackrel{\pi^{\prime}}{\longrightarrow} G(V, r)$. Form following sheaves on the Hilbert scheme $\widetilde{L}_{l}^{h}=\operatorname{det} \pi_{*} \pi^{\prime *} \mathcal{S}(l)$. Since the projection $\pi: \operatorname{Univ}^{P(n)} G(V, r) \rightarrow \operatorname{Hilb}^{P(n)} G(V, r)$ is a flat morphism and sheaves $\mathcal{S}(l)$ are locally free, then sheaves $\widetilde{L}_{l}^{h}$ are invertible.

Proposition 7. [5, proposition 19] Sheaves $\widetilde{L}_{l}^{h}$ are very ample for $l \gg 0$.

Fix the notation $\widetilde{L}_{l}:=\left.\widetilde{L}_{l}^{h}\right|_{H_{0}}$.

We remind the following

Definition 7. [6, definition 4.2.5] Let $Y$ be a k-scheme of finite type, $G$ an algebraic k-group, and $\alpha: Y \times G \rightarrow Y$-group action. G-linearization of a quasicoherent $\mathcal{O}_{Y}$-sheaf $F$ is an isomorphism of $\mathcal{O}_{Y \times G}$-sheaves $\Lambda: \alpha^{*} F \rightarrow p_{1}^{*} F$ where $p_{1}: Y \times G \rightarrow Y$ is the projection and the following cocycle condition holds:

$$
\left(\mathrm{id}_{Y} \times \operatorname{mult}^{*} \Lambda=p_{12}^{*} \Lambda \circ\left(\alpha \times \operatorname{id}_{G}\right)^{*} \Lambda .\right.
$$

Here $p_{12}: Y \times G \times G \rightarrow Y \times G$ is a projection onto first two factors, mult : $G \times G \rightarrow G$ is a morphism of group multiplication in $G$.

Proposition 8. [5, proposition 20] Sheaves $\widetilde{L}_{l}$ carry $G L(V)$-linearization.

Now consider [6, ch. 4, sect. 4.2] an arbitrary one-parameter subgroup $\lambda: \mathbb{A}^{1} \backslash 0 \rightarrow G L(V)$. We denote the image of the point $t \in \mathbb{A}^{1} \backslash 0$ under the morphism $\lambda$ by the symbol $\lambda(t)$. The composite of the morphism $\lambda$ with the action $\alpha$ leads to the morphism $\alpha(\lambda): \mathbb{A}^{1} \backslash 0 \rightarrow \mu(\widetilde{Q})$ for any closed point $\widetilde{x} \in \mu(\widetilde{Q})$. This morphism is given by the correspondence $t \mapsto \widetilde{x}_{t}=\alpha(\lambda(t), \widetilde{x})$.

We claim that the morphism $\alpha(\lambda): \mathbb{A}^{1} \backslash 0 \rightarrow H_{0}$ can be uniquely extended to the morphism $\overline{\alpha(\lambda)}: \mathbb{A}^{1} \rightarrow H_{0}$.

Indeed, existence and uniqueness of the extension to the morphism $\overline{\alpha(\lambda)}$ : $\mathbb{A}^{1} \rightarrow$ Hilb $^{P(n)} G(V, r)$ are provided by properness of the Hilbert scheme. It rests to convince that the point $\overline{\alpha(\lambda)}(0)$ which is added to form the closure of the image, belongs to the subscheme $H_{0}$.

Turn to $G=P G L(V)$-equivariant surjective morphisms

$$
\mu(\widetilde{Q}) \stackrel{\mu^{\prime}}{\longleftarrow} \widetilde{Q}^{\prime} \stackrel{\phi^{\prime}}{\longrightarrow} Q
$$


where $\phi^{\prime}$ is equivariant standard resolution and $\mu^{\prime}$ the morphism to the Hilbert scheme $\operatorname{Hilb}^{P(n)} G(V, r)$.

Let $\widetilde{x}^{\prime}$ be any point in the preimage $\mu^{\prime-1}(\widetilde{x}) \subset \widetilde{Q}^{\prime} ; x=\phi^{\prime}\left(\widetilde{x}^{\prime}\right)$. According to [6, lemma 4.3.8], the morphism $\beta(\lambda): \mathbb{A}^{1} \backslash 0 \rightarrow Q$ defined by the point $x$ is extended to the morphism $\overline{\beta(\lambda)}: \mathbb{A}^{1} \rightarrow Q$. Consider the action $\widehat{\alpha}: G \times \widetilde{Q}^{\prime} \rightarrow \widetilde{Q}^{\prime}$ and an induced morphism $\widehat{\alpha}_{\lambda}:\left(\mathbb{A}^{1} \backslash 0\right) \times \mu^{\prime-1}(\widetilde{x}) \rightarrow \widetilde{Q}^{\prime}$. It includes into the diagram

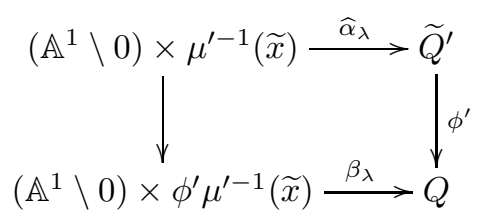

where the lower morphism is induced by the action $\beta: G \times Q \rightarrow Q$. The morphism $\beta_{\lambda}$ extends to the morphism $\bar{\beta}_{\lambda}: \mathbb{A}^{1} \times \phi^{\prime} \mu^{\prime-1}(\widetilde{x}) \rightarrow Q$.

Form a Cartesian square

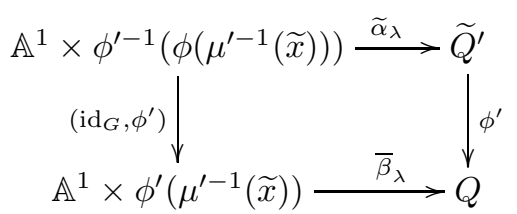

where $\widetilde{\alpha}_{\lambda}$ is a new morphism which is defined by equivariance of the morphism $\phi^{\prime}$ and includes in the commutative diagram

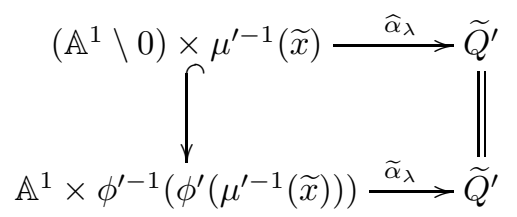

The immersion in this diagram is induced by immersions of factors.

Existence of the morphism $\widetilde{\alpha}_{\lambda}$ points, in particular, on the following fact. The morphism $\widehat{\alpha}(\lambda): \mathbb{A}^{1} \backslash 0 \rightarrow \widetilde{Q}^{\prime}$ defined by any point $\widetilde{x}^{\prime} \in \mu^{\prime-1}(\widetilde{x})$ can be extended to the morphism $\overline{\alpha(\lambda)}: \mathbb{A}^{1} \rightarrow \widetilde{Q}^{\prime}$. Descending relatively to the surjective morphism $\mu^{\prime}$ we come to existence of the extension $\overline{\alpha(\lambda)}: \mathbb{A}^{1} \rightarrow \mu(\widetilde{Q})$ for the morphism $\alpha(\lambda): \mathbb{A}^{1} \backslash 0 \rightarrow \mu(\widetilde{Q})$ defined by an arbitrary point $\widetilde{x} \in \mu(\widetilde{Q})$.

Since $\left(H_{0}\right)_{\text {red }}=\mu(\widetilde{Q})$, the morphism $\alpha(\lambda): \mathbb{A}^{1} \backslash 0 \rightarrow H_{0}$ has a unique extension $\overline{\alpha(\lambda)}: \mathbb{A}^{1} \rightarrow H_{0}$.

Then the point $\widetilde{x}_{0}=\overline{\alpha(\lambda)}(0)$ is a fixpoint of the action of the subgroup $\lambda$. Notation: $\widetilde{x}_{0}=\lim _{t \rightarrow 0} \lambda(t)(\widetilde{x})$. The subgroup $\lambda$ acts on the fibre $L_{\widetilde{x}_{0}}$ of $G$ linearized vector bundle $L$ with some weight $r$. Namely, if $\Lambda$ is the linearization on $L$ then $\Lambda\left(\widetilde{x}_{0}, g\right)=g^{r} \cdot \operatorname{id}_{L_{\widetilde{x}_{0}}}$. Define the weight of the corresponding onedimensional representation of the group $\lambda$ as $w^{\widetilde{L}_{l}}(\widetilde{x}, \lambda)=-r$.

The main tool to analyze the existence of a group quotient is numerical Hilbert - Mumford criterion. Recall 
Definition 8. [6, Definition 4.2.9] The point $x \in X$ of the scheme $X$ is semistable with respect to $G$-linearized ample vector bundle $L$ if there exist an integer $n$ and an invariant global section $s \in H^{0}\left(X, L^{n}\right)$ such that $s(x) \neq 0$. The point $x$ is stable if in addition the stabilizer $\operatorname{Stab}(x)$ is finite and $G$-orbit of the point $x$ is closed in the open set of all semistable points in $X$.

Theorem 3. (Hilbert - Mumford criterion) [6, Theorem 4.2.11] The point $x \in X$ is semistable if and only if for all nontrivial one-parameter subgroups $\lambda: \mathbb{A}^{1} \backslash 0 \rightarrow G$ there is a following inequality

$$
w(x, \lambda) \geq 0 .
$$

The point $x$ is stable if and only if for all $\lambda$ strict inequality holds.

Hilbert - Mumford criterion operates with the set of closed points of the scheme of interest. The set of closed points of $H_{0}$ is the same as the set of closed points in $\mu(\widetilde{Q})$. Hence we can transfer the result of $\S 9[5]$ into our situation.

Theorem 4. [6, theorem 4.2.10] Let $G$ be a reductive group acting on a projective scheme $Y$ with a $G$-linearized ample line bundle $L$. Then there is a projective scheme $X$ and a morphism $f: Y^{s s}(L) \rightarrow X$ such that $f$ is a universal good quotient for the $G$-action. Moreover, there is an open subset $X^{s} \subset X$ such that $Y^{s}(L)=f^{-1}\left(X^{s}\right)$ and such that $f: Y^{s} \rightarrow X^{s}$ is a universal geometric quotient.

We apply this theorem in the following situation: $X=\overline{H_{0}}, G=P G L(V)$, $L=\widetilde{L}_{l}, l \gg 0$. Since we do not know if the equality $\left(\overline{H_{0}}\right)^{s s}=H_{0}$ holds, we have the following proposition.

Proposition 9. There is a quasiprojective algebraic scheme $\widetilde{M}$ with a morphism $f: H_{0}^{s s} \rightarrow \widetilde{M}$, and $f$ is a universal good $P G L(V)$-quotient. The scheme $\widetilde{M}$ contains an open subscheme $\widetilde{M^{s}} \supset \widetilde{M}$ such that the restriction $\left.f\right|_{H_{0}^{s}}: H_{0}^{s} \rightarrow \widetilde{M}$ is a universal geometric quotient.

Proof. Indeed the boundary $\partial \bar{H}_{0}=\bar{H}_{0} \backslash H_{0}$ is $P G L(V)$-invariant subscheme. It is closed in the projective scheme $\bar{H}_{0}$. This implies that $\partial \bar{H}_{0}$ is projective itself. By Theorem 4 the formation of $P G L(V)$-quotients leads to the immersion of projective subscheme $\left(\partial \bar{H}_{0}\right)^{s s} / P G L(V) \hookrightarrow\left(\bar{H}_{0}\right)^{s s} / P G L(V)$ into projective scheme. Then $\widetilde{M}=\left(\left(\bar{H}_{0}\right)^{s s} / P G L(V)\right) \backslash\left(\left(\partial \bar{H}_{0}\right)^{s s} / P G L(V)\right)$ what implies quasi-projectivity of $\widetilde{M}$. The rest of the proposition follows immediately from Theorem 4.

The same is true for the proof that GIT-quotient is indeed moduli space as required: the reasoning of $\S 12[5]$ is transferred in our situation directly.

The GIT-quotient $\widetilde{M}=H_{0}^{s s} / / P G L(V)$ is quasi-projective Noetherian scheme of finite type over $k$. This means that there is a locally closed immersion of the scheme $\widetilde{M}$ into some appropriate projective $k$-space $\mathbb{P}$. Let $j: \widetilde{M} \hookrightarrow \mathbb{P}$ be the corresponding scheme morphism. By [9, chapitre 1, proposition 4.5.15] if $f: X \rightarrow Y$ is open (resp. closed) immersion the same is true for $f_{\text {red }}$. Then 
the reduction $j_{\text {red }}: \widetilde{M}_{\text {red }} \rightarrow \mathbb{P}$ is also locally closed immersion. Now we need an obvious lemma.

Lemma 1. Let $X$ be a projective scheme and $j: X \hookrightarrow \mathbb{P}$ its locally closed immersion into some projective space. Then $j$ is closed immersion.

The application of lemma 1 yields that $\widetilde{M}$ is a projective scheme. The finiteness of the type and Noetherian property descend from the corresponding properties of the scheme $H_{0}$ [10, ch. $1, \S 2$, theorem 1.1].

Proof of the lemma 1. Since $X$ is projective then there is a projective space $\mathbb{P}^{\prime}$ together with closed immersion $j^{\prime}: X \hookrightarrow \mathbb{P}^{\prime}$. Two immersion morphisms $j, j^{\prime}$ induce the morphism $\left(j^{\prime}, j\right): X \hookrightarrow \mathbb{P}^{\prime} \times \mathbb{P}$ of locally closed immersion. We have a commutative diagram

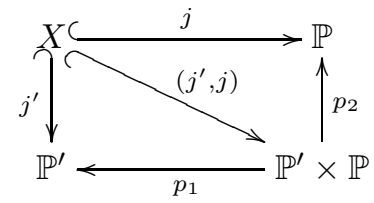

where $p_{1}, p_{2}$ are projections onto the first and the second factor respectively. Representation $j^{\prime}=p_{1} \circ\left(j^{\prime}, j\right)$ guarantees that $\left(j^{\prime}, j\right)$ is closed immersion [9, ch. 1 , remarque 5.2 .7 (iii)]. Then by the representation $j=p_{2} \circ\left(j^{\prime}, j\right)$ we conclude that $j$ is closed immersion.

Remark 1. Taking into account the result of $\S 10$ of the article [5] we come to the following conclusion. The morphism of compactifications constructed there holds when $\widetilde{M}$ and $\bar{M}$ are reduced or when nonreduced moduli schemes $\bar{M}$ and $\widetilde{M}$ are replaced by their reductions. The existence of such a morphism for nonreduced schemes is open question for now.

\section{Subfunctors and moduli subschemes}

Let $\mathfrak{f}:\left(\text { Schemes }_{k}\right)^{o} \rightarrow($ Sets $)$ be a functor assigning to a scheme $T$ the set of families $\mathfrak{F}_{T}$ with base $T$. Families are mentioned to consist of objects of some prescribed class $\mathfrak{F}$. Let $P$ be some property, $\mathfrak{F}^{P}$ subclass of objects in $\mathfrak{F}$ with the property $P . P$ is called open if for any family $\mathfrak{F}_{T}$ points $t \in T$ corresponding to objects with $P$, constitute an open subscheme $T_{0}$ in $T$. Then it makes sense to call the functor $\mathfrak{f}^{P}:\left(\text { Schemes }_{k}\right)^{o} \rightarrow($ Sets $)$ assigning to a scheme $T$ the set of families $\mathfrak{F}_{T}^{P}$ of objects from the class $\mathfrak{F}^{P}$, as open $P$-subfunctor of the functor $\mathfrak{f}$.

Proposition 10. If $\mathfrak{f}$ is corepresented by the scheme $M$ then its open subfunctor $\mathfrak{f}^{P}$ is corepresented by an open subscheme $M_{0}$ in $M$. 
Proof. Corepresentability of the functor $\mathfrak{f}$ by the scheme $M$ means in particular the commutativity of diagrams of the view

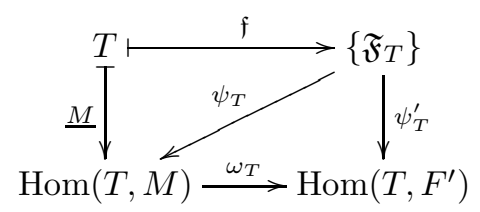

Let $M_{0}$ be the union of images

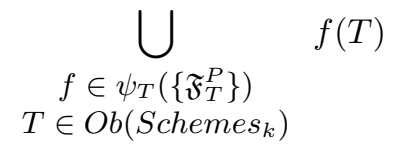

in $M$.

In the sequel when working with the subclass $\mathfrak{F}^{P}$ and with the subfunctor $\mathfrak{f}^{P}$ we use superscript $P$ in notations of natural transformations and induced morphisms in the category of sets: $\psi^{P}, \psi_{T}^{P}$ etc.

It follows from the construction that the subfunctor $\mathfrak{f}^{P}$ is corepresented by the subscheme $M_{0}$. To prove this it is enough to construct a natural transformation $\omega^{P}$. For the scheme immersion $M_{0} \hookrightarrow M$, for each scheme $T$ and for the induced inclusion of sets $\operatorname{Hom}\left(T, M_{0}\right) \hookrightarrow \operatorname{Hom}(T, M)$ consider the diagram

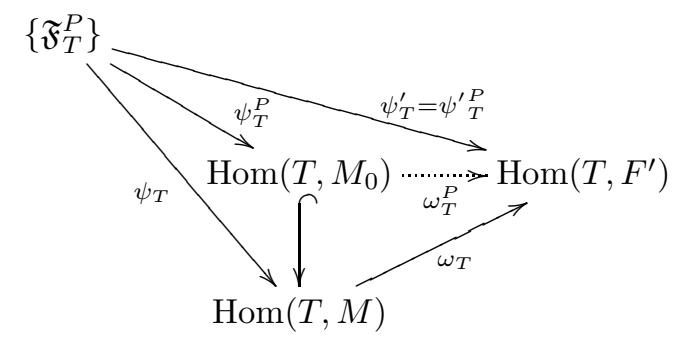

It shows that the map $\omega_{T}^{P}$ is uniquely defined as the composite in the lower triangle. Respectively, for the natural transformation of our interest we have the definition by the composite

$$
\omega^{P}: \operatorname{Hom}\left(-, M_{0}\right) \rightarrow \operatorname{Hom}(-, M) \stackrel{\omega}{\longrightarrow} \operatorname{Hom}\left(-, F^{\prime}\right) .
$$

It rests to convince that $M_{0}$ is open subscheme in $M$. For this consider the covering $M=\bigcup_{U \in O b O p e n(M)} U$ of the scheme $M$ by all possible open subschemes, and corresponding sets $\left\{\mathfrak{F}_{U}\right\}$. Let $U_{0} \subset U$ be the maximal open subscheme in $U$ such that the family $\mathfrak{F}_{U}$ consists of objects with the property $P$ if restricted on $U_{0}$. Such subscheme depends on the choice of family in the set $\left\{\mathfrak{F}_{U}\right\}$. We consider all such subschemes $U_{0}$ for each $U$. It is clear that $\bigcup U_{0} \subset M_{0}$ and $\bigcup U_{0}$ is an open subscheme in $M$.

Now let $T_{0}$ be an arbitrary scheme and let it be the base of a family $\mathfrak{F}_{T_{0}}^{P}$. The natural transformation $\psi$ assigns to it the morphism $f: T_{0} \rightarrow M$. This 
morphism factors through the subscheme $M_{0}$. Then form open covering of the scheme $f\left(T_{0}\right)$ by subschemes $U \times_{M} T_{0}$. Images of these subschemes in $M$ belong to $\bigcup U_{0}$. The consideration of different schemes $T_{0}$ yields in the equality $M_{0}=\bigcup U_{0}$.

Now turn to the class of objects described in (0.1), and to the property $P=\left\{\pi^{-1}(t) \cong S\right\}$. It is clear that $P$ is open property and hence there is open subfunctor $\mathfrak{f}^{P}$. The proposition 10 leads to the existence of the open subscheme $\widetilde{M}_{0}$ which is the coarse moduli space for the subfunctor $\mathfrak{f}^{P}$, i.e. the moduli scheme of $S$-pairs.

Analogously, consider the Gieseker - Maruyama functor $\overline{\mathfrak{f}}:\left(\text { Schemes }_{k}\right)^{o} \rightarrow$ Sets assigning to any scheme $T$ the set of flat $T$-based families $\mathbb{E}$ of semistable coherent torsion-free $\mathcal{O}_{S}$-sheaves $E=\left.\mathbb{E}\right|_{t \times S}$ with Hilbert polynomial $\chi(E \otimes$ $\left.L^{n}\right)=r p_{E}(n)$. The property $P^{\prime}=\{E$ is locally free $\}$ provides the subscheme $\bar{M}_{0}$ of moduli of semistable vector bundles. This subscheme corepresents the subfunctor $\overline{\mathfrak{f}}^{P^{\prime}}$.

By definitions of subfunctors $\mathfrak{f}^{P}$ and $\overline{\mathfrak{f}}^{P^{\prime}}$ they are isomorphic: corresponding classes of objects of parametrization coincide. This implies the isomorphism of moduli schemes $\widetilde{M}_{0} \cong \bar{M}_{0}$. This completes the proof of the theorem 1

Acknowledgement. The author expresses her deep and sincere gratitude to Prof. Dr. Vasile Brinzanescu (IMAR, Bucharest, Romania) for interest to this work and fruitful discussions. Also author thanks the Institute of Mathematics of Romanian Academy (IMAR) where the most part of the work was done, for hospitality and support.

\section{References}

[1] Timofeeva N.V. A compactification of the moduli variety of stable vector 2-bundles on a surface in the Hilbert scheme", Math. Notes. 82:5-6 (2007), $677-690$.

[2] Timofeeva N.V. On a new compactification of the moduli of vector bundles on a surface// Sbornik: Math. 199:7 (2008). P. 1051-1070.

[3] Timofeeva N.V. On a new compactification of the moduli of vector bundles on a surface. II// Sbornik: Math. 200:3 (2009). P. 405-427.

[4] Timofeeva N.V. On degeneration of surface in Fitting compactification of moduli of stable vector bundles// Math. Notes, 90(2011). Also available in arXiv:0809.1148v3.

[5] Timofeeva N.V. On a new compactification of the moduli of vector bundles on a surface. III: Functorial approach// Sbornik: Math. 202:3 (2011). Also available in arXiv:0911.1542.

[6] Huybrechts D., Lehn M. The geometry of moduli spaces of sheaves. Aspects Math., E31. Braunschweig: Vieweg, 1997. 
[7] Maruyama M. Moduli of stable sheaves, II // J. Math. Kyoto Univ. (JMKYAZ) 18-3 (1978). P. 557-614.

[8] A. Bravo, S. Encinas, and O. Villamayor U. A simplified proof of desingularization and applications. Rev. Mat. Iberoamericana 21(2005) $349-458$. arXiv:math/0206244v 2

[9] Grothendieck A., Dieudonné J.A. Eléments de Géométrie Algébrique. I. Springer-Verlag, Berlin - Heidelberg - New York. 1971

[10] Mumford D., Fogarty J. Geometric Invariant Theory. Second enlarged Ed. Springer-Verlag Berlin - Heidelberg - New York, 1982. 